\title{
Measuring impostor phenomenon in healthcare simulation educators: a validation of the Clance Impostor Phenomenon Scale and Leary Impostorism Scale
}

\author{
Kirsty J. Freeman ${ }^{1 *}$, Stephen Houghton ${ }^{2}$, Sandra E. Carr ${ }^{3}$ (D) and Debra Nestel ${ }^{4,5}$ (])
}

\begin{abstract}
Background: Impostor phenomenon is a term used to describe feelings of intellectual and professional fraudulence. The Clance Impostor Phenomenon Scale and the Leary Impostorism Scale are two self-report measures used to determine whether an individual experiences impostor phenomenon. This study examined the psychometric properties of both measures in healthcare simulation educators.

Methods: The study sample comprised 148 educators, 114 (77\%) females, 34 (23\%) males, who completed an online version of each instrument. Exploratory factor analysis was used to examine the factor structure of the Clance Impostor Phenomenon Scale and the Leary Impostorism Scale.

Results: Exploratory factor analysis revealed that for both instruments a one-factor solution best fit the data, suggesting all items in both measures fit onto a single theoretical construct.

Both instruments demonstrated high internal reliability, with the Cronbach's alpha for the Clance Impostor Phenomenon Scale being $a=.96$ and the Leary Impostorism Scale $a=.95$.

Conclusions: This study suggests that impostor phenomenon as measured by the Clance Impostor Phenomenon Scale and the Leary Impostorism Scale is a unidimensional construct among healthcare simulation educators. With a growing interest in impostor phenomenon, the present findings will assist researchers to evaluate the phenomenon in healthcare settings.
\end{abstract}

Keywords: Impostor Phenomenon, Healthcare Simulation Educators, Clance Impostor Phenomenon Scale, Leary Impostor Scale, Exploratory Factors Analysis

\section{Background}

Described as an individual's persistent belief that they lack intelligence, skills or competence, and are not worthy of success, [1-3] the term impostor phenomenon (IP) was first introduced by Clance and Imes [2]. Their study of 150 highly successful women found that "despite

*Correspondence: Kirsty.Freeman@duke-nus.edu.sg

${ }^{1}$ Duke NUS Medical School, Singapore, Singapore

Full list of author information is available at the end of the article outstanding academic and professional accomplishments, women who experience the impostor phenomenon persist in believing that they are really not bright and have fooled anyone who thinks otherwise" [2]. Over the last 40 years research has shown that IP is experienced by individuals irrespective of gender, profession or cultural background [4-8].

Harvey and Katz suggested that nearly $70 \%$ of successful people have experienced IP in their working life [3]. Studies of IP in healthcare professionals has been focused 
on those entering the workforce for the first time, [9, 10] with only a few studies investigating the phenomenon in the current workforce [11-13]. The existence and impact of IP in the healthcare simulation educators is unknown. IP exists on a continuum from occasional feelings of inadequacy to constant feelings of being exposed as a fraud [1]. The more frequently IP is experienced the greater the likelihood of negative effects. IP has been reported to negatively impact job satisfaction, [14] increase emotional exhaustion, [15] and lead to burnout [16]. To enable faculty developers to mitigate the negative impact of IP in the healthcare simulation educator community we must first investigate the prevalence.

The increasing level of interest in IP has seen the development of several self-report instruments to measure IP, including the Clance Impostor Phenomenon Scale (CIPS), [17] Harvey Impostor Scale, [18] Perceived Fraudulence Scale, [19] and Leary Impostorism Scale (LIS) [20]. The LIS is a 7 item unidimensional instrument measuring a person's sense of being an impostor or fraud [20]. The authors have reported high inter-item reliability $(\alpha=0.87)$. The 20 item CIPS is reported to be the most commonly used scale by those researching IP, with Cronbach alphas ranging from 0.85 to 0.96 [21]. Chrisman et al. reported that the CIPS comprises three factors - luck, fake and discount [22]. Brauer and Wolf also reported three factors in their EFA of the German version of the CIPS [23]. Subsequent studies suggest one, two and three-factor models best explain the factor structure of the instrument $[6,7,24,25]$. For researchers investigating IP in healthcare simulation educators the use of an instrument that has been validated within a related context is important. This together with the limited number of studies reporting the use of the 7-item LIS offers a clear rationale for further examination and validation of the instrument. The aim of this study is to examine the psychometric properties of the CIPS and LIS, and provide evidence for their validity within the healthcare simulation population.

\section{Method}

\section{Participants and procedure}

The sample comprised 148 healthcare simulation educators, $114(77 \%)$ females and $34(23 \%)$ males. Of the respondents, 86 (58\%) were aged 40 to 55 years, 37 (25\%) 56 to 74 years and 25 (17\%) 24 to 39 years. Responses were received from respondents in nine countries, with the majority currently working in the United States of America (61\%), followed by Australia (22\%), and United Kingdom (7\%), with Canada, Denmark, Portugal, Singapore, Thailand and Turkey comprising the remaining $10 \%$.
The study was approved by the Human Ethics Research Office of The University of Western Australia (RA/4/20/5061). An invitation to participate in the study was distributed via the international simulation community through SimConnect, the online community platform of the Society for Simulation in Healthcare, based in the USA $(n=4000)$; the Australian based National Health Education and Training in Simulation (NHET-Sim) community $(n=5000)$; and the WA Simulation in Healthcare Alliance $(n=20)$, based in Western Australia. A cover letter informed all potential respondents their participation was voluntary and that responses were anonymous. Those who agreed to take part were given a link to an online form, where they were introduced to the research objectives. They were then provided with instructions on completing the anonymous questionnaires, subject to confirming their informed consent. All respondents provided demographic information, such as age, gender, and geographical location, along with other information. They then completed the CIPS and the LIS. No incentives were provided.

\section{Measurement of impostor phenomenon}

The psychometric properties of the CIPS and LIS were examined by establishing the respective factor structures from a sample of 148 healthcare simulation educators. An Exploratory Factor Analysis (EFA) was employed to establish which latent variables optimally summarised the observed variables on each of the measures.

\section{Analyses}

Prior to performing the EFA, the suitability of the data for factor analysis was assessed on the basis on three criteria: (a) a visual inspection of the data matrix for correlations in excess of 0.30, [26] (b) Bartlett's test of sphericity [27] and (c) Kaiser-Meyer-Olkin's measure of sampling adequacy [28]. After assessing the suitability, the 20 items of the CIPS, and the 7 items of the LIS were subjected to maximum likelihood (ML) factor analysis with (orthogonal) varimax rotation. According to Kline, loadings of 0.3 and above are regarded as significant with a minimum sample of 100 participants [29]. The number of factors to be retained for interpretation were then determined by six criteria: 1) Kaiser-Guttman's eigenvalue of greater than 1.0 rule; 2) Cattell's scree test; 3) Horn's parallel analysis; 4) the cumulative percentage of variance criterion; 5) the evaluation of factor loading patterns; and 6) the interpretability criterion of factor loading [30-34]. Analyses were conducted using IBM SPSS version 27. 


\section{Results \\ CIPS}

The correlation matrix revealed the presence of a substantial number of correlation coefficients above 0.3, indicating some underlying relationship among the variables and therefore the suitability of the correlation matrix for factor analysis. Bartlett's test of sphericity [22] $\left(x^{2}=2232, d f=190, p<0.001\right)$, supported the factorability of the matrix, while Kaiser-Meyer-Olkin's measure of sampling adequacy [28] (0.95), suggested sufficient common variance among the observed variables for factor analysis. Descriptive statistics for the CIPS are shown in Table 1.

A maximum likelihood (ML) factor analysis with (orthogonal) varimax was conducted. ML factor analysis extracts a set of factors by successive factoring, each of which in turn explains as much variance as possible in the population correlation matrix, as estimated from the sample correlation matrix [29]. ML provides statistical significance testing of each factor as it is extracted, [34] and varimax is the preferred method of rotation when the research goal is item reduction and where data will be subsequently used in multivariate analysis [33].
An unrestricted ML factor analysis with varimax rotation revealed the presence of two possible factors with eigenvalues exceeding 1.0, explaining 55.69\% and $5.76 \%$, of the variance, respectively. The screeplot suggested a probable one-factor solution in comparison with the two-factor solution derived with the eigenvalue rule. The first factor comprised of 12 items and the second 8 items. As shown in Table 2, of the 20 items, 11 cross loaded with values on both factors greater than 0.39 , with some having cross loadings on both factors exceeding this.

Given the cross-loadings the number of factors to extract was then manually specified at three and four, but in each case the factor solution consisted of multiple cross loadings, items not loading on any factor, and a single item factor being derived. In each of these analyses the iterative removal of items did not resolve the problems.

The factor analysis was re-run with one factor specified and this revealed a one factor solution, explaining $55.69 \%$, of the variance. As can be seen in Table 3 all variables had loadings above 0.30 and all loaded on the single factor.

Table 1 Descriptive statistics and CITC of the CIPS 20 items $(N=148)$

\begin{tabular}{|c|c|c|c|}
\hline CIPS Items & Mean & SD & CITC \\
\hline 1-I have often succeeded on a test or task even though I was afraid that I would not do well before I undertook the task & 3.52 & 1.13 & .58 \\
\hline 2-I can give the impression that I'm more competent than I really am & 2.98 & 1.17 & .61 \\
\hline 3-I avoid evaluations if possible and have a dread of others evaluating me & 2.26 & 1.05 & .49 \\
\hline $\begin{array}{l}\text { 4-When people praise me for something I've accomplished, I'm afraid I won't be able to live up to their expectations of me in the } \\
\text { future }\end{array}$ & 2.70 & 1.21 & .82 \\
\hline $\begin{array}{l}\text { 5-I sometimes think I obtained my present position or gained my present success because I happened to be in the right place at } \\
\text { the right time or knew the right people }\end{array}$ & 2.97 & 1.45 & .73 \\
\hline 6-I'm afraid people important to me may find out that I'm not as capable as they think I am & 2.53 & 1.27 & .81 \\
\hline 7-I tend to remember the incidents in which I have not done my best more than those times I have done my best & 3.24 & 1.26 & .73 \\
\hline 8 - I rarely do a project or task as well as l'd like to do it & 2.71 & 1.21 & .66 \\
\hline 9-Sometimes I feel or believe that my success in my life or in my job has been the result of some kind of error & 2.00 & 1.19 & .75 \\
\hline 10-It's hard for me to accept compliments or praise about my intelligence or accomplishments & 3.26 & 1.10 & .68 \\
\hline 11-At times, I feel my success has been due to some kind of luck & 2.43 & 1.19 & .77 \\
\hline 12-I'm disappointed at times in my present accomplishments and think I should have accomplished much more & 2.98 & 1.18 & .59 \\
\hline 13-Sometimes I'm afraid others will discover how much knowledge or ability I really lack & 2.43 & 1.15 & .82 \\
\hline 14-I'm often afraid that I may fail at a new assignment or undertaking even though I generally do well at what I attempt & 2.79 & 1.14 & .81 \\
\hline $\begin{array}{l}\text { 15-When I've succeeded at something and received recognition for my accomplishments, I have doubts that I can keep repeat- } \\
\text { ing that success }\end{array}$ & 2.55 & 1.18 & .84 \\
\hline $\begin{array}{l}\text { 16-If I receive a great deal of praise and recognition for something I've accomplished, I tend to discount the importance of what } \\
\text { I've done }\end{array}$ & 3.20 & 1.17 & .71 \\
\hline 17-I often compare my ability to those around me and think they may be more intelligent than I am & 3.06 & 1.32 & .75 \\
\hline $\begin{array}{l}\text { 18-I often worry about not succeeding with a project or examination, even though others around me have considerable confi- } \\
\text { dence that I will do well }\end{array}$ & 2.94 & 1.22 & .79 \\
\hline 19-If I'm going to receive a promotion or gain recognition of some kind, I hesitate to tell others until it is an accomplished fact & 3.60 & 1.20 & .63 \\
\hline 20-I feel bad and discouraged if I'm not "the best" or at least "very special" in situations that involve achievement & 2.91 & 1.23 & .59 \\
\hline
\end{tabular}

Response options ranged from $1=$ not at all true to $5=$ very true. CITC - corrected item-total correlations; CIPS =Clance Impostor Phenomenon Scale 
Table 2 Unrestricted factor-loading matrix

\begin{tabular}{|c|c|c|}
\hline & \multicolumn{2}{|c|}{ Factor } \\
\hline & 1 & 2 \\
\hline \multicolumn{3}{|c|}{ CIPS Items } \\
\hline Q18 & .856 & .301 \\
\hline Q14 & .783 & .389 \\
\hline Q17 & .731 & .361 \\
\hline Q15 & .681 & .539 \\
\hline Q19 & .585 & .293 \\
\hline Q12 & .551 & .306 \\
\hline Q1 & .533 & .293 \\
\hline Q7 & .529 & .511 \\
\hline Q16 & .527 & .475 \\
\hline Q10 & .521 & .450 \\
\hline Q8 & .510 & .439 \\
\hline Q20 & .501 & .332 \\
\hline Q11 & .348 & .793 \\
\hline Q9 & .321 & .784 \\
\hline Q5 & .317 & .755 \\
\hline Q6 & .456 & .744 \\
\hline Q13 & .519 & .695 \\
\hline Q4 & .571 & .606 \\
\hline Q2 & .336 & .550 \\
\hline Q3 & .334 & .371 \\
\hline
\end{tabular}

Loadings highlighted in bold indicate the factor on which the item was placed

Table 3 One factor specified factor-loading matrix

\begin{tabular}{ll}
\hline CIPS ITEMS & \\
\hline Q15 & .867 \\
Q13 & .856 \\
Q6 & .844 \\
Q4 & .836 \\
Q14 & .823 \\
Q18 & .808 \\
Q11 & .795 \\
Q9 & .772 \\
Q17 & .770 \\
Q5 & .749 \\
Q7 & .735 \\
Q16 & .715 \\
Q10 & .693 \\
Q8 & .673 \\
Q2 & .627 \\
Q19 & .625 \\
Q12 & .607 \\
Q20 & .592 \\
Q1 & .588 \\
Q3 & .502 \\
\hline Exran Met &
\end{tabular}

Extraction Method: Maximum Likelihood
A parallel analysis [32] was conducted with a randomly generated data set (20 variables, $N=148,100$ to 1000 replications was run) using a Monte Carlo PCA for parallel analysis [34]. The results indicated that for one factor, the eigenvalues from the sample in this study (11.138) exceeded the corresponding eigenvalues (PCA $\max =1.73 ; 100$ to 1,000 replications). This further suggests that all items on the CIPS were assessing one underlying dimension.

The cumulative percent of the one-factor solution of the 20 CIPS items accounted for $55.69 \%$, which is above the minimum level of $30 \%$. The interpretability of the items and factor loadings was impostor phenomenon. Cronbach's alpha for the CIPS was $\alpha=0.96$, indicating a high degree of internal consistency.

\section{The LIS}

The same EFA procedure used with the CIPS was applied to the seven items of the LIS. The correlation matrix revealed the presence of a substantial number of correlation coefficients above 0.3. Bartlett's test $\left(\chi^{2}=885\right.$, $d f=21, p<0.001)$ supported the factorability of the correlation matrix, while the MSA (0.91) suggested sufficient common variance among the observed variables for factor analysis.

An unrestricted maximum likelihood (ML) factor analysis with (orthogonal) varimax rotation revealed the presence of one possible factor with an eigenvalue exceeding 1.0 , and explaining $73.4 \%$ of the variance. Table 4 shows the mean and standard deviation for each of the items.

An inspection of the screeplot for the LIS revealed a probable one-factor solution and the cumulative percent of the one-factor solution of the seven LIS items accounted for $73.4 \%$ of the variance. All items had loadings above 0.30 and all loaded on the single factor (see Table 5). Item 7 cross-loaded but removal of this item did not improve the factor solution or the reliability of the scale that comprised distinctively of items measuring impostor phenomenon.

A parallel analysis (7 variables, $N=148,50$ to 1000 replications conducted) indicated that for one factor, the eigenvalue (5.138) from the sample in this study exceeded the corresponding eigenvalues ( $\mathrm{PCA} \max =1.323 ; 50$ to 1,000 replications) obtained from the randomly generated data set of the same size. Cronbach's alpha for the LIS was $\alpha=0.94$, suggesting that it has a high level of internal consistency. This indicates that the LIS functioned as a relatively homogeneous scale in this sample.

With the EFA supporting a one-factor solution for both instruments, a Pearson correlation coefficient was conducted to assess the relationship between the total scores on the LIS and the CIPS. There was a significant positive 
Table 4 Descriptive statistics for the 7 item LIS and CITC ( $N=148)$

\begin{tabular}{|c|c|c|c|}
\hline Item & Mean & SD & $\begin{array}{l}\text { Corrected } \\
\text { Item-Total } \\
\text { Correlation }\end{array}$ \\
\hline 1-Sometimes I am afraid I will be discovered for who I really am & 2.17 & 1.097 & .826 \\
\hline 2-I tend to feel like a phony & 1.99 & 1.085 & .837 \\
\hline 3-I'm afraid people important to me may find out that I'm not as capable as they think I am & 2.39 & 1.248 & .845 \\
\hline 4-In some situations I feel like an imposter & 2.47 & 1.209 & .817 \\
\hline 5-Sometimes I'm afraid others will discover how much knowledge or ability I really lack & 2.39 & 1.187 & .864 \\
\hline 6-In some situations I feel like a "great pretender"; that is, I'm not as genuine as others think I am & 2.03 & 1.112 & .819 \\
\hline 7-In some situations I act like an imposter & 1.74 & .971 & .591 \\
\hline
\end{tabular}

Response options ranged from $1=$ is not characteristic at all of me to $5=$ extremely of me. CITC corrected item-total correlations

Table 5 Unrestricted factor-loading matrix

\begin{tabular}{lll}
\hline & \multicolumn{2}{l}{ Factor } \\
\cline { 2 - 3 } & $\mathbf{1}$ & $\mathbf{2}$ \\
\hline Q1 & $\mathbf{. 9 1 1}$ & -.122 \\
Q2 & $\mathbf{. 8 9 8}$ & -.195 \\
Q3 & $\mathbf{. 8 5 4}$ & .023 \\
Q4 & $\mathbf{. 8 5 1}$ & -.038 \\
Q5 & $\mathbf{. 8 4 6}$ & .304 \\
Q6 & $\mathbf{. 8 3 9}$ & .030 \\
Q7 & $\mathbf{. 6 1 0}$ & .370 \\
\hline
\end{tabular}

Extraction Method: Maximum Likelihood

correlation between the total scores of the measures, $r=0.828, n=148, p=<0.001$.

\section{Discussion}

The CIPS and LIS are two instruments frequently used to measure the construct of impostor phenomenon, the former being the most popular. Previous studies have reported inconsistent findings, however, with regard to their psychometric properties. Therefore, this present study sought to establish their factorial structure and internal reliability.

The EFAs conducted supported a one factor model for both the 20-items CIPS and the 7-items LIS. Both instruments also produced high internal reliabilities. Previous studies have reported one, two, three and four factor solutions identifying subscales such as luck, fear, fake, and discount. When confirmatory factor analysis has been used for the CIPS, only 16 items have been included and it has been unclear which items load onto the factors. Mak et al. recently noted that the CIPS does not measure subscale characteristics [21] Therefore, optimum factor structures have not been clear. By conducting a rigorous EFA the present findings indicate both measures are unidimensional, which is consistent with Jöstl et al., [7] and Simon and Choi, [24] who report a single factor best explains the structure of the CIPS. Similarly, previous research shows the 7 item LIS is also a unidimensional measure of impostorism [35]. A significant positive correlation was established between the total scores of the CIPS and LIS, suggesting that researchers investigating IP in healthcare simulation educators could utilise the shorter 7-item LIS.

\section{Conclusion}

This study suggests that impostor phenomenon, as measured by the CIPS and LIS, is a unidimensional construct, and that both the CIPS and LIS have sound psychometric properties for measuring IP among healthcare simulation educators. With the increasing interest in IP globally, these findings will increase the scientific community's confidence in measuring the construct using CIPS and LIS.

\section{Abbreviations}

IP: Impostor phenomenon; CIPS: Clance Impostor Phenomenon Scale; LIS: Leary Impostorism Scale; EFA: Exploratory Factor Analysis; ML: Maximum likelihood; MSA: Measurement statistical analysis.

\section{Acknowledgements}

The authors would like to thank Ms. Pauline Clance for the permission to use the Clance Impostor Phenomenon Scale.

\section{Authors' contributions}

$\mathrm{KF}, \mathrm{SH}, \mathrm{SC}$, and DN made a substantial contribution to the rationale and design of the study. KF and $\mathrm{SH}$ analysed the data, interpreted the results and drafted the manuscript. All authors commented on each draft of the manuscript. All authors read and approved the final manuscript.

\section{Funding}

None.

Availability of data and materials

The datasets used and/or analysed during the current study are available from the corresponding author on reasonable request. 


\section{Declarations}

\section{Ethics approval and consent to participate}

Ethics approval was granted by The University of Western Australia Human Research Ethics Committee: HREC RA/4/20/5061, with the study conducted in accordance with the National Statement on Ethical Conduct in Human Research. All participants provided written informed consent prior to participating in the study.

\section{Consent for publication}

Not applicable.

\section{Competing interests}

The authors declare that they have no competing interests.

\section{Author details}

${ }^{1}$ Duke NUS Medical School, Singapore, Singapore. ${ }^{2}$ Graduate School of Education, University of Western Australia, Perth, Australia. ${ }^{3}$ Health Professions Education, School of Allied Health, The University of Western Australia, Perth, Australia. ${ }^{4}$ School of Clinical Sciences, Monash University, Melbourne, Australia.

${ }^{5}$ Department of Surgery, University of Melbourne, Melbourne, Australia.

Received: 23 September 2021 Accepted: 21 February 2022

Published online: 03 March 2022

\section{References}

1. Hibberd J. The Imposter Cure: How to stop feeling like a fraud and escape the mind-trap of imposter syndrome. Great Britan: Aster; 2019.

2. Clance PR, Imes SA. The imposter phenomenon in high achieving women: dynamics and therapeutic intervention. Psychother: Theory Res Pract. 1978;15(3):241-7.

3. Harvey J, Katz C. If I'm so successful why do i feel like a fake? New York: St. Martin's Press; 1985.

4. Cozzarelli C, Major B. Exloring the validity of the impostor phenomenon. J Soc Clin Psychol. 1990;9(4):401-17.

5. Holmes SW, Kertay L, Adamson LB, Holland CL, Clance PR. Measuring the impostor phenomenon - a comparison of Clance IP scale and Harvey I-P scale. J Pers Assess. 1993;60(1):48-59.

6. French BF, Ullrich-French SC, Follman D. The psychometric properties of the Clance Impostor Scale. Personality Individ Differ. 2008;44(5):1270-8.

7. Jöstl G, Bergsmann E, Lüftenegger M, Schober B, Spiel C. When Will They Blow My Cover? The Impostor Phenomenon Among Austrian Doctoral Students. Zeitschrift für Psychologie. 2012;220(2):109-20.

8. Landry MJ, Bailey DA, Ervin A. You Are Not an Impostor: The Registered Dietitian Nutritionist and Impostor Phenomenon. J Nutr Educ Behav. 2021:53(7):625-30.

9. Aubeeluck A, Stacey G, Stupple EJN. Do graduate entry nursing student's experience 'Imposter Phenomenon'?: An issue for debate. Nurse Educ Pract. 2016;19:104-6.

10. Henning K, Ey S, Shaw D. Perfectionism, the impostor phenomenon and psychological adjustment in medical, dental, nursing and pharmacy students. Med Educ. 1998;32(5):456-64.

11. Gottlieb M, Chung A, Battaglioli N, Sebok-Syer SS, Kalantari A. Impostor syndrome among physicians and physicians in training: A scoping review. Med Educ. 2019:54(2):116-24.

12. Hutchins $H$, Penney L, Sublett L. What imposters risk at work; exploring imposter phenomenon, stress coping, and job outcomes. Hum Resour Dev Q. 2018;29:31-48.

13. Legassie J, Zibrowski EM, Goldszmidt MA. Measuring Resident Well-Being: Impostorism and Burnout Syndrome in Residency. Journal of general internal medicine : JGIM. 2008;23(7):1090-4.

14. Vergauwe J, Wille B, Feys M, De Fruyt F, Anseel F. Fear of being exposed: The trait-relatedness of the impostor phenomenon and its relevance in the work context. J Bus Psychol. 2015;30(3):565-81.

15. Neureiter $M$, Traut-Mattausch $E$. Inspecting the Dangers of Feeling like a Fake: An Empirical Investigation of the Impostor Phenomenon in the World of Work. Front Psychol. 2016;7:1445.
16. Hutchins HM. Outing the Imposter: A Study Exploring Imposter Phenomenon among Higher Education Faculty. New Horizons in Adult Education and Human Resource Development. 2015;27(2):3-12.

17. Clance PR. The Impostor Phenomenon: when success makes you feel like a fake. Toronto: Bantam Books; 1985.

18. Harvey JC 1981 The impostor phenomenon and achievement: a failure to internalize success. Available from ProQuest Dissertations \& Theses Global. https://www.proquest.com/dissertations-theses/impostor-pheno menon-achievement-failure/docview/303035505/se-2?accountid= 14681.

19. Kolligian J Jr, Sternberg RJ. Perceived Fraudulence in Young Adults: Is There an "Imposter Syndrome"? J Pers Assess. 1991;56(2):308-26.

20. Leary MR, Patton KM, Orlando AE, Wagoner FW. The Impostor Phenomenon: Self-Perceptions, Reflected Appraisals, and Interpersonal Strategies. J Pers. 2000;68(4):725-56.

21. Mak KKL, Kleitman S, Abbott MJ. Impostor Phenomenon Measurement Scales: A Systematic Review. Front Psychol. 2019;10:671.

22. Chrisman SM, Pieper WA, Clance PR, Holland CL, Glickaufhughes C. Validation of the clance impostor phenomenon scale. J Pers Assess. 1995:65(3):456-67.

23. Brauer K, Wolf A. Validation of the German-language Clance Impostor Phenomenon Scale (GCIPS). Personality Individ Differ. 2016;102:153-8.

24. Choi SM, Y-J,. Using factor analysis to validate the Clance Impostor Phenomenon Scale in sample of science, technology, engineering and mathematics doctoral students. Personality Individ Differ. 2018;121:173-5.

25. Yaffe Y. Validation of the Clance Impostor Phenomenon Scale with female Hebrew-speaking students. J Exp Psychopathol. 2020;11(4):2043808720974341.

26. Tabachnick BG, Fidell LS. Using multivariate statistics. 6th ed. Boston: Pearson Education; 2013.

27. Bartlett MS. A note on the multiplying factors for various chi square approximation. J R Stat Soc. 1954;16(Series B):296-8.

28. Kaiser H. An index of factorial simplicity. Psychometrika. 1974;39:31-6.

29. Kline RB. Principles and Practice of Structural Equation Modeling, Fourth Edition. 4th ed. New York: Guilford Publications; 2015.

30. Guttman L."Best possible" systematic estimates of communalities. Psychometrika. 1956;21:273-85.

31. Cattell RB. The Scree Test For The Number Of Factors. Multivar Behav Res. 1966;1 (2):245-76

32. Horn JL. A rationale and test for the number of factors in factor analysis. Psychometrika. 1965;30:179-85.

33. Hair JF, Black WC, Babin BJ, Anderson RE. Multivariate data analysis. Seventh edition, Pearson new international. edition. Harlow: Pearson Education Limited; 2014.

34. Watkins MW. Exploratory Factor Analysis: A Guide to Best Practice. J Black Psychol. 2018;44(3):219-46.

35. Ibrahim F, Münscher JC, Herzberg PY. The facets of an impostor - development and validation of the impostor-profile (IPP31) for measuring impostor phenomenon. Curr Psychol. 2020. https://doi.org/10.1007/ s12144-020-00895-x.

\section{Publisher's Note}

Springer Nature remains neutral with regard to jurisdictional claims in published maps and institutional affiliations. 